\section{Un nouvel acteur dans la perception gustative des lipides alimentaires: le canal calcique TRPC3}

Amira Sayed Khan, Aziz Hichami, Naim Akhtar Khan
Inserm U1231, Équipe Physiologie

de la nutrition et toxicologie,

université Bourgogne Franche-Comté( UBFC),

6 boulevard Gabriel,

21000 Dijon, France.

naim.khan@u-bourgogne.fr
> Le sens du goût (gustation) joue un rôle clé dans le choix des aliments. II existe cinq modalités gustatives: amer, salé, acide, sucré, et umami ${ }^{1}[1]$. Le goût sucré permet la détection de substances énergétiques riches en calories, tandis que le goût amer permet d'éviter ou de rejeter les substances toxiques ou nocives. Ces deux modalités gustatives sont bien conservées phylogénétiquement. Les hommes et les rongeurs manifestent une préférence spontanée pour les lipides alimentaires. Les résultats d'études récentes suggèrent qu'il pourrait exister une sixième modalité gustative pour la détection orosensorielle des acides gras alimentaires à longue chaîne (AGLC) [2]. Nous avons montré que la préférence pour les AGLC alimentaires est modifiée chez les individus obèses, hommes et animaux, et que ce phénomène est associé à l'altération des mécanismes de la perception des lipides [3]. Nous avons identifié deux récepteurs des lipides (CD36 et GPR120 [G-protein-coupled receptor 120]), situés dans la partie apicale des bourgeons du goût, et qui sont impliqués respectivement dans la détection sensorielle orale des AGLC et dans le contrôle post-ingestif de la prise alimentaire [3]. Par ailleurs, notre équipe a confirmé, dans des populations tunisienne, algérienne et tchèque d'obèses, qu'un polymorphisme génétique de CD36

\footnotetext{
1 L'umami, mot japonais signifiant «délicieux, savoureux », correspond au goût du mono-glutamate de sodium, un goût plaisant, proche du sucré.
}

réduisant l'expression de cette protéine augmente le seuil de détection orosensorielle des AGLC alimentaires chez les sujets obèses, et pourrait par conséquent expliquer leur consommation excessive de lipides [4].

\section{Signalisation gustative des lipides}

Nous avons montré que l'interaction d'un AGLC avec son récepteur gustatif active la phospholipase C (PLC), qui catalyse l'hydrolyse du phosphatidyl-inositol-4,5-biphosphate $\left(\mathrm{PIP}_{2}\right)$ en inositol-1,4,5-triphosphate $\left(\mathrm{IP}_{3}\right)$ et diacylglycérol (DAG). $\mathrm{L}^{\prime} I P_{3}$, en se liant à ses récepteurs dans la membrane du réticulum endoplasmique, libère des ions $\mathrm{Ca}^{2+}$ dans le cytoplasme, ce qui contribue à l'augmentation de la concentration intracellulaire de $\mathrm{Ca}^{2+}\left(\left[\mathrm{Ca}^{2+}\right]_{\mathrm{i}}\right)$, suivie d'un efflux puis d'un influx d'ions $\mathrm{Ca}^{2+}$ via l'ouverture de canaux calciques, composés de protéines ORAI (aussi appelées calcium releaseactivated calcium channel proteins). Ces canaux sont soit homomériques, comme ORAIl, soit hétéromériques, comme ORAII/3 [3-6]. Ils sont activés seulement lorsque la réserve d'ions $\mathrm{Ca}^{2+} \mathrm{du}$ réticulum endoplasmique est mobilisée, et appartiennent donc à la famille des canaux SOC (store-operated calcium). L'ouverture des canaux SOC est sous le contrôle de la protéine STIMI (stromal interaction molecule 1 ), qui détecte la quantité d'ions $\mathrm{Ca}^{2+}$ nécessaire pour remplir le réticulum endoplasmique $[6,7]$. Restait à savoir si le DAG était, comme I'IP $P^{3}$, impliqué dans la signalisation gustative des lipides.
Des canaux TRPC sont responsables de l'influx d'ions $\mathrm{Ca}^{2+}$ lors de la perception gustative des lipides Les canaux SOC incluent vraisemblablement aussi des canaux de la famille TRP (transient receptor potential). Les canaux TRP sont regroupés en 7 familles, en fonction de leurs homologies de séquence d'acides aminés: TRPC, TRPM, TRPV, TRPA, TRPP, TRPML et TRPN. Chaque sous-unité d'un canal TRP est constituée de six domaines transmembranaires, avec un pore situé entre le cinquième et le sixième segment transmembranaires, une structure semblable à celle des canaux potassiques sensibles au potentiel de membrane [8]. L'activité de la plupart des canaux de la famille TRPC (TRP « canoniques ») est modulée par les acides gras, sous forme libre ou estérifiée en position médiane ( $s n-2)$ du glycérol du DAG. Tous les canaux TRPC (TRPC1, TRPC2, TRPC3/6/7 et TRPC4/5) peuvent être activés en réponse à la stimulation de récepteurs couplés aux protéines G (G-protein-coupled receptors, GPCR), dont font partie les récepteurs de la vision, de l'olfaction et de la gustation. Ils sont activés par la PLC via I'hydrolyse de $\mathrm{PIP}_{2}$ en $\mathrm{IP}_{3}$ et $\mathrm{DAG}$, mais alors que les canaux TRPC $3 / 6 / 7$ sont plus spécifiquement activés par le $D A G$, les canaux TRPCl/4/5 ne le sont pas, et leur mécanisme d'activation par la PLC reste controversé [8]. Nous avons montré que les cellules gustatives fongiformes de la souris expriment les canaux TRPC3 (mais pas TRPC6 ou TRPC7), et que ces canaux sont aussi sous le contrôle 


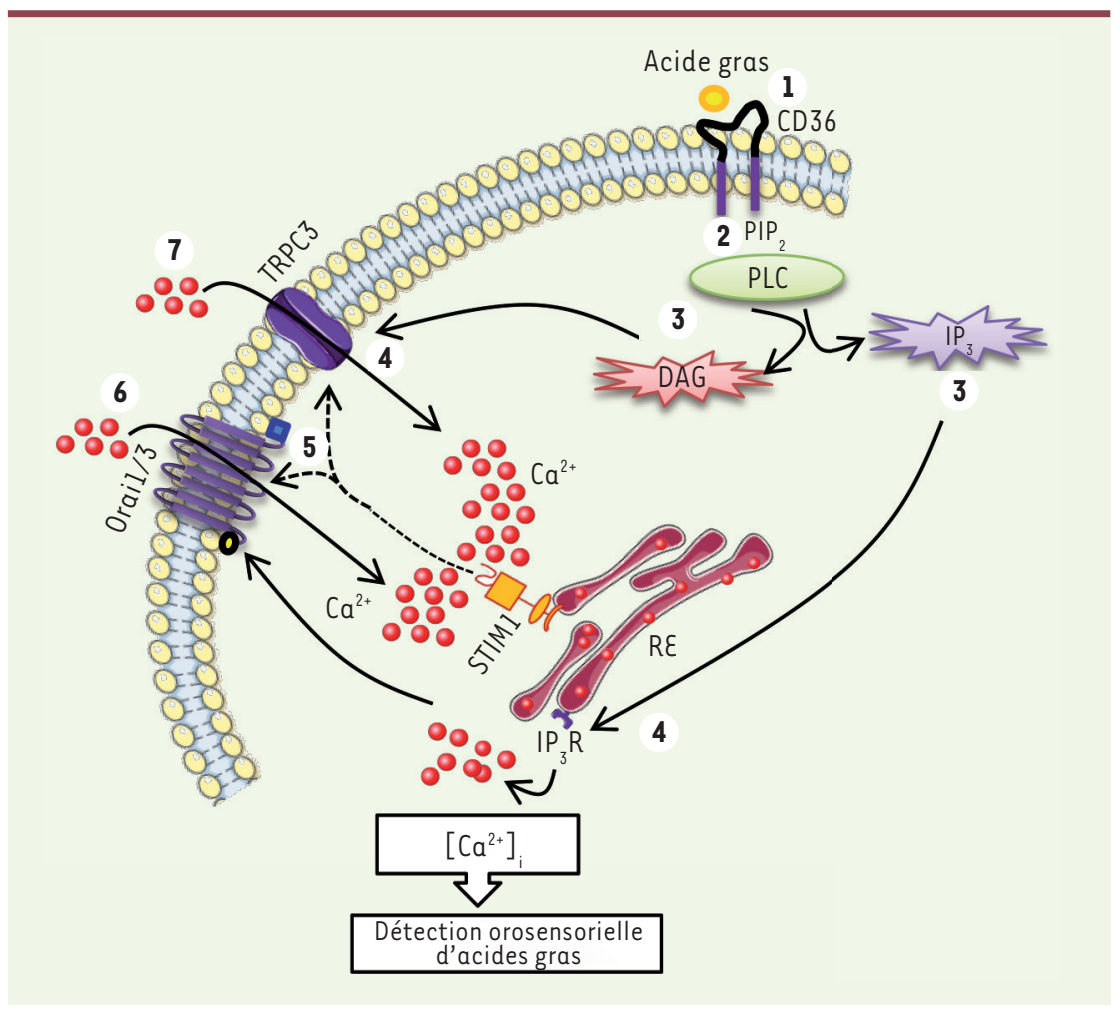

de STIMI, comme le sont les canaux calciques ORAIl et ORAII/3 [9] (Figure 1). Dans ces cellules, l'acide linoléique (un acide gras alimentaire à longue chaîne [AGLC]), en se liant au lipido-récepteur CD36, induit la production de DAG d'une manière PLC-dépendante. Une analyse par spectroscopie de masse a permis de montrer que cet $A G L C$ exogène s'incorpore dans le diacylglycérol (DAG) en position médiane du glycérol (sn-2), produisant du 1-stéaroyl-2-linoléoylsn-glycérol (SLG) [10].

Afin de prouver l'implication physiologique de TRPC3 dans la perception gustative des AGLC, nous avons analysé le comportement alimentaire de souris dont les gènes TRPC3 (Transient Receptor Potential Cation Channel Subfamily C Member 3), TRPC6, ou TRPC7 ont été invalidés. Alors que les souris témoins ont une préférence alimentaire pour l'acide linoléique, cette préférence était plus faible chez les souris mutantes $\operatorname{Trpc} 3^{-} /^{-}$(mais pas chez les souris $\operatorname{Trpc6} \%^{-} /^{-}$ou $\operatorname{Trpc} 7^{-} /^{-}$). Une diminution de la préférence pour l'huile de colza a également été observée chez ces souris [9]. Enfin, nous avons montré que l'activation des canaux TRPC3 des cellules sensorielles gustatives par les AGLC est couplée à des mécanismes physiologiques d'anticipation de la prise alimentaire, en observant, après l'application d'un AGLC sur la partie apicale de la langue, que la libération de deux peptides anorexigènes intestinaux (cholécystokinine et peptide yy) dans le sang (via le circuit neuronal langue-cerveau-intestin) était diminuée chez les souris $\operatorname{Trpc}^{-} /^{-}$par rapport à des souris témoins [9]. $\diamond$

TRPC3 calcium channel: A new chainlink in the gustatory perception of dietary lipids

\section{LIENS D'INTÉRÊT}

Les auteurs déclarent n'avoir aucun lien d'intérêt concernant les données publiées dans cet article.

\section{RÉFÉRENCES}

1. Chaudhari N, Roper SD. The cell biology of taste. J Cell Biol 2010 ; 190: 285-96.

2. Besnard P, Passilly-Degrace P, Khan NA. Taste of fat: a sixth taste modality? Physiol Rev 2016 ; 96: 151-76.

3. Khan AS, Keast R, Khan NA. Preference for dietary fat: from detection to disease. Prog Lipid Res 2020 ; 78 : 101032.

4. Khan AS, Murtaza B, Hichami A, Khan NA. A cross-talk between fat and bitter taste modalities. Biochimie 2019 ; 159: 3-8
Figure 1. Implication des canaux TRPC3 dans la détection orosensorielle des acides gras alimentaires. La liaison d'un acide gras à la protéine CD36 de la membrane plasmique (1) active la phospholipase C (PLC) (2), qui hydrolyse le phosphatidyl inositol-4,5-biphosphate $\left(\mathrm{PIP}_{2}\right)$ en inositol-1,4,5-triphosphate $\left(I_{3}\right)$ et diacylglycérol (DAG) (3). La liaison de $I$ 'I $P_{3}$ à son récepteur $\left(I P_{3} R\right)$ dans $l a$ membrane du réticulum endoplasmique $(R \varepsilon)$ entraîne la libération intracellulaire d'ions $\mathrm{Ca}^{2+}$, tandis que le DAG active les canaux TRPC3, ce qui contribue aussi à l'augmentation de la concentration intracellulaire de $\mathrm{Ca}^{2+}\left(\left[\mathrm{Ca}^{2+}\right]_{\mathrm{i}}\right)$ (4). La libération de $\mathrm{Ca}^{2+}$ dans le cytoplasme par l'IP $P_{3}$ est détectée par STIMl, qui active les canaux ORAII/3 et TRPC3 présents dans la membrane plasmique (5), et provoque l'augmentation prolongée de $\left[\mathrm{Ca}^{2+}\right]_{\mathrm{i}}$ (6 et 7 ) nécessaire à la transmission nerveuse du message orogustatif vers le cerveau.

5. Gilbertson TA, Khan NA. Cell signaling mechanisms of oro-gustatory detection of dietary fat: advances and challenges. Prog Lipid Res 2014 ; 53: 82-92.

6. Dramane G, Abdoul-Azize S, Hichami A, et al. STIMI regulates calcium signaling in taste bud cells and preference for fat in mice. J Clin Invest 2012 ; 122: 2267-82.

7. Ozdener MH, Subramaniam $S$, Sundaresan $S$, et al. CD36- and GPR120-mediated $\mathrm{Ca}^{2+}$ signaling in human taste bud cells mediates differential responses to fatty acids and is altered in obese mice. Gastroenterology 2014 ; 146: 995-1005

8. Chen X, Sooch G, Demaree IS, et al. Transient receptor potential canonical (TRPC) channels: then and now. Cells 2020 ; 9: 1983.

9. Murtaza B, Hichami A, Khan AS, et al. Implication of TRPC3 channel in gustatory perception of dietary lipids. Acta Physiol (Oxf) 2021 ; 231: el3554.

10. Madani S, Hichami A, Legrand A, et al. Implication of acyl chain of diacylglycerols in activation of different isoforms of protein kinase C. FASEB J 2001 ; 15: 2595-601.
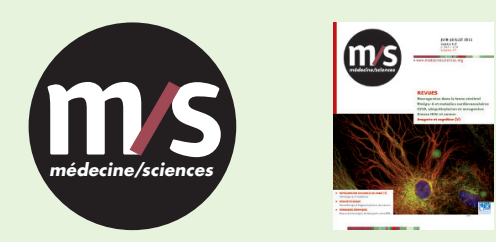

Abonnez-vous

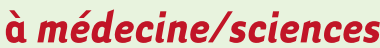

Bulletin d'abonnement page 806 dans ce numéro de $\mathrm{m} / \mathrm{s}$ 